\title{
Design and Experimentation of a Rover Concept for Lunar Crater Resource Survey
}

\author{
David Wettergreen, Dominic Jonak, David Kohanbash, \\ Scott Moreland, Spencer Spiker, James Teza, and William Whittaker \\ Carnegie Mellon University, 5000 Forbes Avenue. Pittsburgh, PA 15213 \\ dsw@ri.cmu.edu
}

\begin{abstract}
Scarab is a prospecting rover for lunar missions to survey resources, particularly water ice, in polar craters. It is designed for the deployment of a deep coring drill and for transport of soil analysis instruments. Its chassis can transform to stabilize the drill in contact with the ground and can also adjust to ascend and descent steep slopes of unconsolidated soil. Additional features include a compact body for better thermal regulation, laser scanners for dark navigation, and power system designed for a persistent, low-capacity source. Scarab was prototyped at the Robotics Institute, has undergone mobility testing in soils laboratories and field sites leading up to an integrated system test including the RESOLVE drill and instrument suite at the PISCES lunar analogue site on Mauna Kea in Hawaii.
\end{abstract}

\section{Introduction}

To discover and measure the resources of the moon and to someday extract oxygen, hydrogen and possibly water from lunar regolith, robotic systems will have to survive brutal extremes from blazing sunlight to frigid darkness as well as dust, vacuum, and isolation. Discovering water ice in significant abundance on the lunar surface would transform our approach to a sustainable human presence on the moon. The Lunar Prospector orbiter measured greater hydrogen concentrations in the permanently shadowed craters on the lunar poles than in sunlight areas [Spudis06], this discovery fuels the desire to explore the lunar poles in the search for hydrogen, water ice, and other resources. To explore these resource-rich sites, machines will need to operate in darkness, survive extreme cold, and be able to negotiate steep slopes and crated terrain.

Scarab is a prospecting rover developed to perform the necessary science operations to locate volatiles and validate In Situ Resource Utilization (ISRU) methods. (Figure 1.) It is terrestrial concept vehicle designed to deploy a deep coring drill and to transport soil analysis instruments. The vehicle design employs a passive kinematic suspension with active adjustability to lower for drilling and aid in

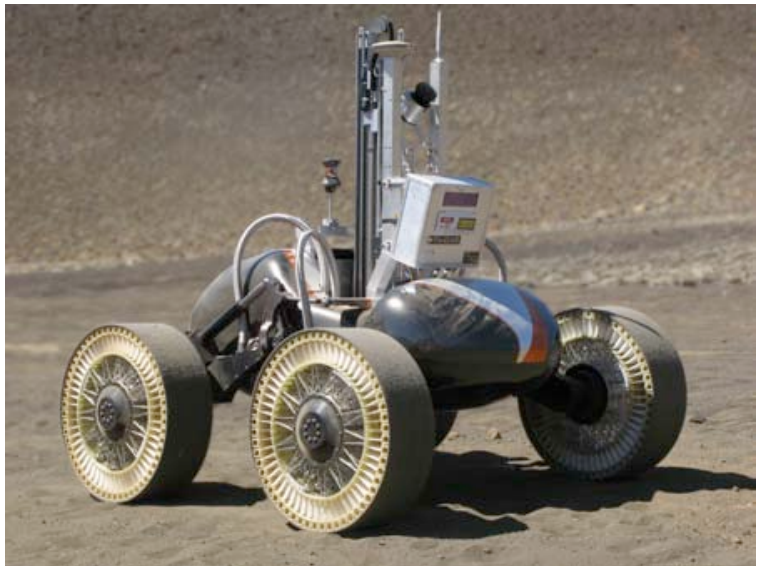

Figure 1. Scarab rover with RESOLVE drill and instrument payload on Mauna Kea, Hawaii

driving. Its chassis can transform to stabilize the drill in contact with the ground and can also adjust to ascend and descent steep slopes of unconsolidated soil. Scarab can control roll, meaning rotation about its longitudinal axis, by independently adjusting its side-frames and this allows it to drive cross-slope. turn switchbacks, and ascend and descend loose materials.

Scarab uses an onboard inertial measurement device and ground speed sensors to enable it to estimate position and velocity in the dark. Laser range scanners are used to build models of the surrounding terrain to detect obstacles and then determine efficient and safe paths.

Scarab transports a coring drill and sample recovery mechanism developed by the NORthern Center for Advanced Technology (NORCAT). This mechanism captures pristine one-meter core samples and crushes core segments for analysis by science instruments. [Norcat08]

Scarab carries science instruments to perform autonomous in situ processing of core samples. This payload is called RESOLVE for Regolith and Environment Science and Oxygen and Lunar Volatile Extraction and has been developed by NASA ISRU program by Glenn Research Center, Kennedy Space Center and Johnson Space Center. It includes a thermal reactor, gas absorption beds, a gas 
chromatograph, as well as other instruments and associated valving and control. [Kleinhenz09] [Muscatello09] After the sample is acquired from the sample recovery unit, it is analyzed for volatiles of interest, with a focus on hydrogen bearing molecules. This is followed by extracting the trapped water molecules from the sample and performing an electrolysis to obtain hydrogen and oxygen.

In this paper we present a description of the mechanical, electrical, and software design of prototype rover. Then we review experimental results from field experiments on Mauna Kea, Hawaii verify its capability to meet the needs of the polar crater survey mission.

\section{Design}

There are many factors involved in the design of the Scarab rover but the drill mechanism and operation dominated the design. The requirement to transport and stabilize the drill literally became central to the rover design. At the same time, meeting requirements for ascent and descent in cratered terrain shaped many aspects and fine details.

\subsection{Considerations}

Scrab is concieved as work machine with a serialized mission: drive, charge batteries, drill, charge, process samples, charge and repeat. The number of repetitions might be 25 , leading to 25 kilometers of traverse, 25 cores, and 25 sites surveyed. For some craters, 100 repetitions might be more desirable to characterize the environment and resources.

The rationale for the vehicle weight and size class is based on the $\sim 1 \mathrm{~m}$ long, $\sim 3 \mathrm{~cm}$ diameter drill class that is likely to be employed in such a mission. Not only does the rover have to package the drill within it but also it must provide sufficient weight on the lunar surface against which the drill can react its downward thrust and torque about the bit. Drill thrusts could be expected to reach $250 \mathrm{~N}$ and $50 \mathrm{Nm}$ torque. The system weight on lunar surface must react drilling $250 \mathrm{~N}$ downforce and maintain $150 \mathrm{~N}$ on wheels for stability against uplift and spin, therefore total weight on lunar surface must be greater than $400 \mathrm{~N}$. The weight in lunar gravity $\left(400 \mathrm{~N} / 1.622 \mathrm{~m} / \mathrm{s}^{2}=250 \mathrm{~kg}\right)$ leads to a vehicle mass of $250 \mathrm{~kg}$. [Bartlett08]

Drilling requires a stiff platform for reliable operation, into which thrust loads, torques and vibrations are transmitted. Placement of the drill in line with the vehicle's center of mass maximizes the mass that can be applied to drill down force. (Figure 2) Affixing directly to a high stiffness, high strength chassis maximizes the stability of the platform. A fixed drill structure configuration was chosen due to the stiffness and reduced actuations, and since the vertical structure can also support navigation sensors. This dual purpose comes from the need for the drill to be upright when the vehicle is at a coring site, and for a navigation mast to be upright when the vehicle is driving. A flight implementation may require a

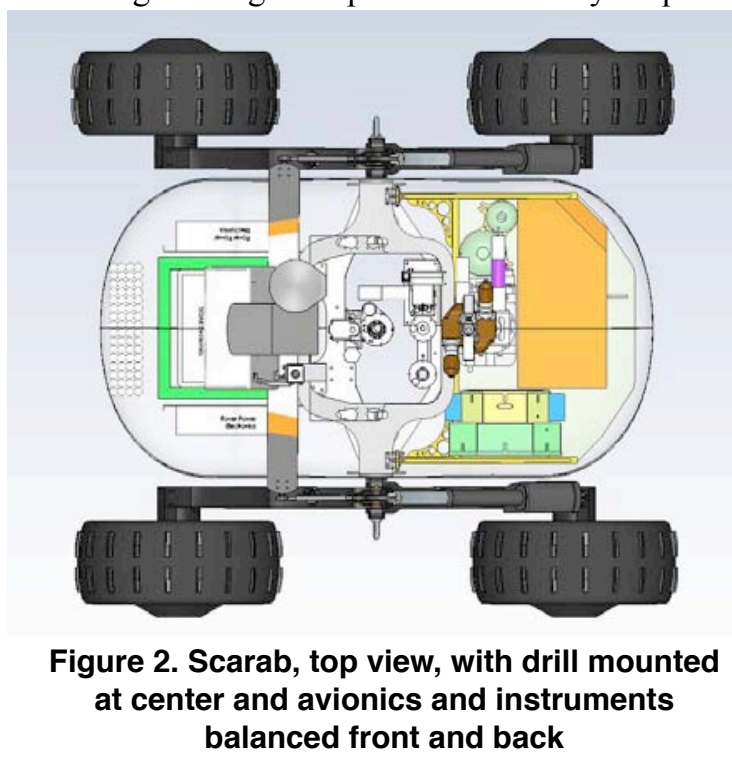

single use deployment actuation depending on stowing requirements.

The design considerations for mobility were to achieve a low center of mass for stability, to use skid steering as opposed to explicit steering, and to employ an adjustable kinematic suspension. Skid steering is favored due to the simplicity it affords the system and the precedence for it on the Soviet Lunokhod rovers.

A passive, differenced rocker suspension matches the wheels to high roughness terrain, evenly distributes weight among the four wheels, and creates a large rectangular base for the vehicle's stability pyramid.

\subsection{Description}

\subsubsection{Chassis and Suspension}

The Scarab lunar rover's chassis and suspension was designed primarily around the drill sampling system. This component of the payload is significant in mass and imposes large forces on the rover during transport and while reacting with the ground.

As with all space systems, reducing mass is vital because of the cost of launch is function of the mass to be lifted. A unique constraint was placed on this rover system is that there is also a minimum mass in order to provide enough weight to support the drilling thrust. The lunar mission calls for a minimum design mass of approximately $250 \mathrm{~kg}$ to react drill thrust, while reserving weight for traction to resist torques. 
To better utilize the little traction available, the drill core and rover center-of-mass are collocated and the Scarab suspension was designed to actively lower its chassis and extend its wheel base. Drilling operations receive three benefits of this feature; first, lowering the chassis allows the full stroke of the 1 meter core to be utilized resulting in mass saving. Secondly, when operating on slopes, the rover can 'lean' and therefore re-stabilize and place the rover center-ofmass over the drill core. Lastly, under low gravity conditions, the drill torques are counteracted by the

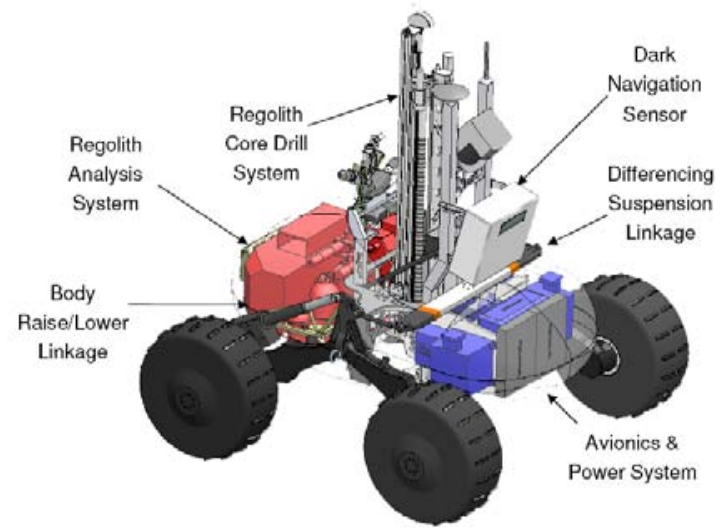

Figure 3. Scarab rover configuration showing placement of sensors, avionics, and payload. There are drive motors in each of four wheels and two linkages for adjusting sideframe height. An averaging linkage allows all four wheels to maintain ground contact in rough terrain.

increased leverage arm created by spreading the rover wheelbase.

The Scarab rover suspension system has active and passive elements for increased traction on difficult terrain. The active element, as previously discussed with respect to drilling, allows the rover to level the body, leading to increased stability and traction efficiency increases. The passive element, sometimes called "body differencing or averaging" provides a mechanical release allowing the two rover suspension side frames to pivot independently. (Figure 3.) As the center-of-mass of the rover is located midway between the side frames, equal wheel loading results in all four wheels even on drastically uneven terrain.

Scarab's chassis allows it to passively conform to the terrain. The differencing linkage ensures the body is pitch-averaged between the rocker arms. Scarab's body has three contact points. On either side, the body is connected to the pivot in the rocker arms. On top, the body hangs from the differencing linkage. This linkage runs across the top of the body and also connects to the rocker arms. Scarab actively controls its roll using the rocker arms. The rocker arms allow
Scarab to change the height of each side independently, thus controlling the roll. In contrast the pitch is passive, Scarab can not actively control this. Scarab's wheels conform to the terrain, which rotates the rocker arms and swivels the differencing linkage. The linkage is constructed such that the body is forced to move up or down to be exactly the average of the two rocker arms.

\subsubsection{Payload}

The RESOLVE payload, which had been fully developed as a laboratory prototype, was configured to fit within Scarab while simultaneously Scarab was modified to meet the structural, thermal, and access requirements of the payload. The design mass for RESOLVE which includes the drill, sample transfer and preparation, and all instrumentation is $100 \mathrm{~kg}$. Modifications were made to the initial Scarab rolling chassis to position avionics and rover power in the front of the chassis and allow maximum volume for instruments in the rear. The RESOLVE payload required control electronics, amplifiers, power and gas reserves that were not onboard the rover. They are connected only when sample acquisition and processing is underway. The payload is designed to be removable for calibration and service. (Figure 4.)

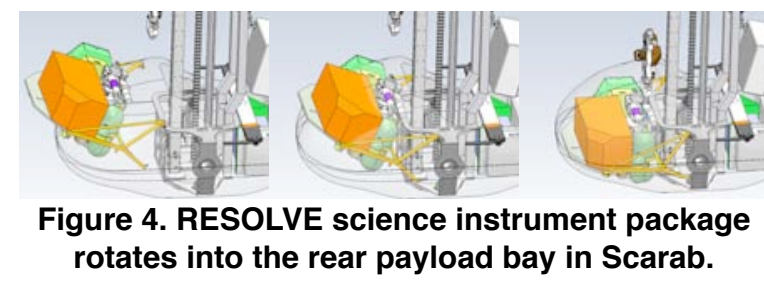

The most significant design consideration in accommodating the RESOLVE payload is that the mass of the body must be balanced between the front and back of the rover in order to minimize the load on the differencing suspension linkage. If one end is significantly higher in mass the body will pitch in that direction. In this prototype an almost 50-50 mass balance was achieved largely by placing batteries in the extreme front of the rover and by leaning sensors forward from the mast.

\subsubsection{Power}

Scarab is powered by two 28 volt lithium ion batteries which provide 26 amp hour capacity allowing operation for three hours of continuous topspeed locomotion or six hours of typical activity. Each battery feeds the main power bus via steering diodes so that one battery can be removed for external charging with interrupting operation. Each battery can be charged on board Scarab using 


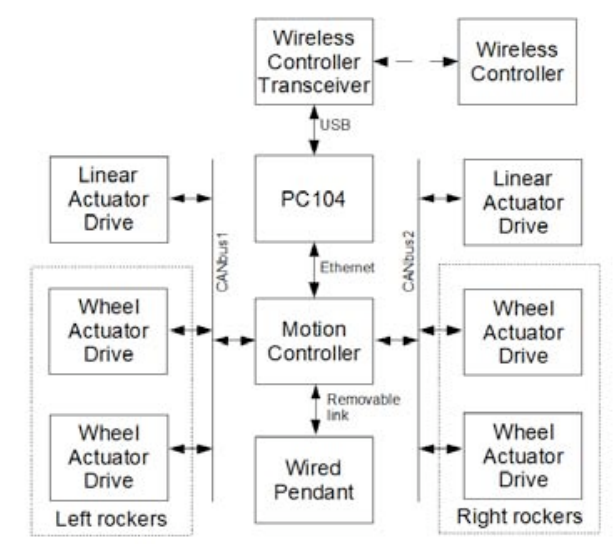

Figure 5. Avionics configuration with motor control units, amplifiers and drivetrains embedded within the rockers.

independent charging systems powered externally via a tether from alternating current produced by either a portable generator or mains. Integrated charge controllers in each of the batteries perform charge balancing and provide telemetry describing battery state to the on board computer via a serial data interface.

DC/DC converters (Vicor VI-x00 types) furnish regulated DC power to the various computing, control and sensing systems on board Scarab, however payload power is not provided. The wheel actuator amplifiers draw on a 48 volts DC bus powered by a boost converter developed for Scarab by NASA Glenn Research Center. In addition, this converter provides telemetry describing input and output bus voltages and currents.

\subsubsection{Locomotion}

The locomotion system consists of four in hub motors with associated mechanical reduction and two linear actuators which actuate the pose adjustment mechanisms of the side frames. The in hub wheel motors are DC brushless type (Kollmorgen AKM series) which drive combined planetary and harmonic drive reducers (80:1 HD Systems CFG unit with 2:1 input planetary) to yield total 160:1 reduction. The sealed linear actuator is driven by a brushed DC motor (SKF Runner series) modified by addition of a string potentiometer to provide position feedback.

The electronics of the locomotion system consists of a dedicated motion controller (Elmo Maestro) and individual motor amplifiers (Elmo Harmonica series) for each of the six actuators. The controller communicates to each of the amplifiers via two CAN bus networks, one for each side of the robot, as shown in figure 5. The controller is located in the main body of scarab and the amplifiers for each of the wheel actuators are located within the respective rocker arm assemblies, thus reducing cabling needed to pass from any of the rocker arms to the main body and utilizing volume of the hollow rocker arms. Communication between the controller and the PC-104 on-board computer is via Ethernet. Software running in the controller provides several modes of operation - low level control giving direct control of actuation or pass through of commands from a high level controller running on the on-board computer. Direct control of the locomotion system is via a removable wired pendant providing individual control of left and right wheel velocities or pose adjustment without requiring use of the on board computer. A wireless hand controller for the locomotion system is available through a USB connection to the on-board computer.

\section{Table 1. Vehicle specifications}

Drill tower (upright): $2.2 \mathrm{~m}$ high stance,

\section{$1.6 \mathrm{~m}$ low stance}

Mass: $280 \mathrm{~kg}$

Weight: $2740 \mathrm{~N}$ Earth surface

$450 \mathrm{~N}$ Lunar surface

Locomotion speed: $3-6 \mathrm{~cm} / \mathrm{s}$

Wheel diameter: $65 \mathrm{~cm}$

Track width: $1.4 \mathrm{~m}$

Wheelbase: $0.8-1.4 \mathrm{~m}$

$1.2 \mathrm{~m}$ nominal

Aspect ratio: 1:1.0 low stance

(track/wheelbase) 1:1.2 nominal stance

$1: 1.7$ high stance

CG planar location: On geometric center

CG height: $0.48 \mathrm{~m}$ low stance

$0.64 \mathrm{~m}$ nominal stance

$0.74 \mathrm{~m}$ high stance

Static pitch-over: $56^{\circ}$ low stance

$43^{\circ}$ nominal stance

$30^{\circ}$ high stance

Static roll-over: $61^{\circ}$ low stance

$53^{\circ}$ nominal stance

$49^{\circ}$ high stance

Maximum straddle: $55 \mathrm{~cm}$

Minimum straddle: Belly contact

\section{Experiments}

Testing the Scarab rover has been critical in proving the concept for lunar mobility and drilling and quantifying performance of the system. Experiments have been conducted in a few conditions with several initial findings of importance. However it is understood that further experimentation is needed to provide the data for a fully validated performance model and, most important, to enable extrapolation of terrestrial results to the lunar environment. 
Initial characterization of the Scarab rover mobility system was undertaken at Carnegie Mellon and at NASA Glenn Research Center's SLOPE Facility. Field experiments were performed in order to characterize not only the rover performance, but the specifics of the terrain that is trafficable.

The SLOPE (Simulated Lunar Operations) facility provided an opportunity to quantify the effectiveness of active body leveling (center of mass control) while cross-sloping. (Figure 6.) Tests were conducted on a hydraulically adjustable slope. In addition to the cross-slope experiments, drawbar pull tests gathering load/slip curves were recorded.

Moses Lake Sand Dunes in Washington State was chosen as a test site for its varied terrain (slopes, craters, etc), low moisture content, varied soil types (strengths, size distribution) and wide open space. These qualities provided grounds for mobility traction testing and long distance dark navigation traverses. Steep slope ascent/descent in loose soil and the test of multiple slope climbing techniques where the focus of these field tests

The PISCES (Pacific International space Center for Exploration Systems) lunar analogue site located on Mauna Kea, Hawaii is at high altitude with dry, deep, basaltic volcanic ash. The experiments conducted on Mauna Kea included testing the full Scarab rover system with its ISRU core drill sampling and analysis payload. The soil composition and mechanical properties at this site were ideal for the regolith sampling hardware experiments. The objectives of these tests were to demonstrate roving, drilling, sample acquisition, processing and analysis. The rover was capable of autonomously traversing kilometers of rough terrain, inspecting a drill site, drill to 1 meter depth, process the core samples and analyze the composition of the capture and demonstrate production of water taken from the sample. Secondary to these tests, quantitative analysis of newly developed flight relevant lunar wheels were performed with Scarab.

\subsection{Traction}

Characterization of the Scarab rover as a system for difficult terrain mobility was first quantified at the SLOPE facility. The independently actuated rocker arms of the Scarab rover allow for actively controlled center-of-mass shifting. Benefits of this feature include decreased slip during cross-slope maneuvers among others.

The Scarab rover was commanded to cross-slope $10^{\circ}, 15^{\circ}$ and $20^{\circ}$ inclines of GRC- 1 simulant at the SLOPE facility. A robotically controlled total station was used to track a $360^{\circ}$ prism mounted on the rover. This allowed for highly accurate position and instantaneous slip measurements to be recorded. The

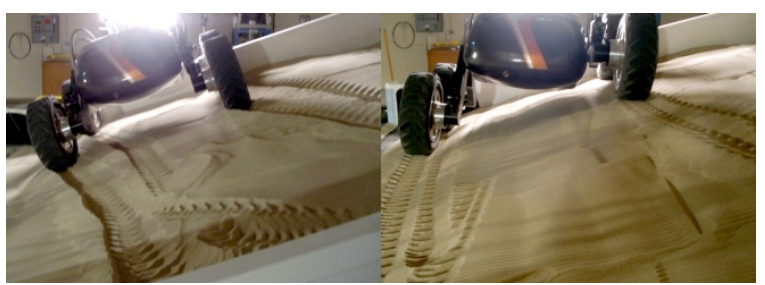

Figure 6. Conventional and Roll Control CrossSlope Driving showing differences in downhill slip

outcomes of these tests, shown below, are expressed as percentage downhill slip with respect to crossslope distance in Table 2.

Table 2. Downhill Slip

\begin{tabular}{|l|l|l|l|}
\hline $\begin{array}{l}\text { Slope } \\
\text { Angle }\end{array}$ & Conventional & $\begin{array}{l}\text { Body Roll } \\
\text { Controlled }\end{array}$ & $\begin{array}{l}\text { Slip } \\
\text { Difference }\end{array}$ \\
\hline $10^{\circ}$ & $6 \%$ & $2 \%$ & $-4 \%$ \\
\hline $15^{\circ}$ & $22 \%$ & $8 \%$ & $-14 \%$ \\
\hline $20^{\circ}$ & $37 \%$ & $15 \%$ & $-22 \%$ \\
\hline
\end{tabular}

The considerable decrease in downhill slip $(2.5 \mathrm{x}$ at $20^{\circ}$ incline) arises from increased traction due to equalization of wheel loading in highly cohesive soils and edging effects of the wheel profile in frictional soils. The significance of this outcome lies in the ability to descend and navigate steeper slopes with while maintaining adequate control authority.

A widely used metric for measuring the total tractive ability of a system (rover or vehicle) is called drawbar pull. This essentially is the value the vehicle can pull in a specified material while maintaining forward progress. The drawbar pull value corresponds to the knee of the load/slip curve where the soil fails and the wheel enters high slip regime. This value is quite informative when comparing different designs but also used for determining the slope a vehicle can ascend for the specified material.

Drawbar Pull experiments were conducted at GRC SLOPE facility, Moses Lake Washington and Mauna Kea Hawaii. This allowed for tests in seven unique material types including basaltic volcanic soil and lunar simulant. (Table 3.)

The drawbar pull tests conducted allowed for the evaluation of the effects of rover mass properties, wheel design, and soil properties on tractive performance. Both the drawbar pull value and power values derived from this test are used as metrics to determine performance. 
Table 3. Pull and Power versus Soil Types

\begin{tabular}{|l|l|l|l|}
\hline Variable & Site & $\begin{array}{l}\text { Drawbar } \\
\text { Pull }\end{array}$ & $\begin{array}{l}\text { Nominal } \\
\text { Drive } \\
\text { Power }\end{array}$ \\
\hline $\begin{array}{l}\text { Sand, Loose, } \\
\text { GRC1 }\end{array}$ & GRC SLOPE & $27 \%$ & $120 \mathrm{~W}$ \\
\hline $\begin{array}{l}\text { Sand, Compact, } \\
\text { GRC1 }\end{array}$ & GRC SLOPE & $30 \%$ & $103 \mathrm{~W}$ \\
\hline Sand, Best 530 & GRC SLOPE & $30 \%$ & $105 \mathrm{~W}$ \\
\hline $\begin{array}{l}\text { Sand, Loose, } \\
\text { Natural }\end{array}$ & Moses Lake & $20 \%$ & $134 \mathrm{~W}$ \\
\hline Basalt, Fine & Mauna Kea & $28 \%$ & $158 \mathrm{~W}$ \\
\hline $\begin{array}{l}\text { Add Mass (25\%) } \\
\text { Add Depth (to }\end{array}$ & Mauna Kea & $39 \%$ & $103 \mathrm{~W}$ \\
\hline $\begin{array}{l}\text { 2.5cm) } \\
\text { Change Wheel }\end{array}$ & Mauna Kea & $23 \%$ & $100 \mathrm{~W}$ \\
\hline
\end{tabular}

The key points to take from these tests are the range of tractive values that occur with changing properties. For high bearing strength materials such as GRC-1, the level of looseness and compaction does result in varied traction or power (shear strength and sinkage respectively). The overall mass (even loading of wheels) also has little effect on the normalized drawbar pull value (percentage of vehicle weight). It should be noted with extremely low bearing strength materials, this does not hold true as a result of excessive sinkage. The shear strength of comes from its cohesiveness and internal friction. As a result, the drawbar pull values can be representations of slopes achievable for only highly cohesive material as the normal loading of the surface is constant throughout testing. The most significant effect on traction has resulted in wheel design. Experiments involving different traction surfaces, wheel diameter and ground pressures have shown a large range of drawbar pull values. Differences of $50 \%$ have been achievable through traction surface/grouser modifications. Lowering ground pressure and reducing sinkage has moderate effects on traction but results in large differences in driving power (up to $50 \%$ during experiments).

Drawbar pull tests performed as laboratory and field experiments have highlighted wheel design as a leading element in tractive and power design requirements. This is an important realization as wheel design is generally independent of the mobility system suspension design and can be optimized for traction and power efficiencies.

Active control methods can also lead to increased tractive performance. Techniques such as "inchworming" and actively controlled center-of-mass shifting can greatly increase the mobility of a rover. (Figure 7.)
To begin the cycle of inch-worming on the Scarab rover, the body lowers while expanding the wheelbase and rolling the front wheel forward while the rear wheel remains static. For the second half of the cycle, the body raises and the wheel base shortens while the rear wheel rolls forward and the front wheel remains static. It is these static wheels that provide the benefits of inch-worming. To achieve these benefits, Scarab's inch-worming routine relies on eliminating the compaction resistances on two of the four wheels (by keeping static with respect to ground) for a resulting net tractive increase of the mobility system, thus allowing the rover to maneuver over steeper terrain and looser soils.

During field experimentation it has been demonstrated that the inch-worming technique is best suited when wheels become entrenched under high slip. It allows the rover to move forward or back up out of this difficult situation.

\subsection{Steep Slope Ascent}

Although inch-worming, as described in the previous section, can increase mobility on difficult terrain and slopes, actively controlled center of mass shifting can greatly increase steepness of slopes traversable.

The ability to redistribute the loading amongst the rover's four wheels leads to more efficient traction. This center of mass shifting or 'leaning' control of Scarab was field tested at both Moses Lake Sand Dunes and the Mauna Kea analogue site. Evaluation of this technique was performed under the metric of "heading slip." This is the measure of slip in the direction the rover was commanded to head with respect to the commanded velocity. Results from the Moses Lake and Mauna Kea field tests are shown in Table 4.

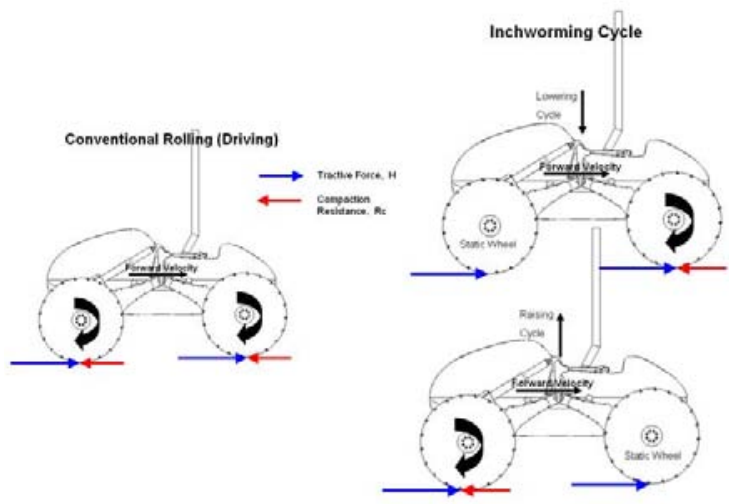

Figure 7. Conventional rolling with all wheels turning in unison versus inch-worming where one turning wheel is synchronized to the side-arm expansion/contraction and one fixed wheel reacts forces into the ground. 


\begin{tabular}{|c|c|c|c|c|}
\hline \multicolumn{2}{|c|}{ Table 4. Performance } & \multicolumn{3}{|c|}{$\begin{array}{l}\text { Slip Ratio on Commanded } \\
\text { Velocity along Ascent }\end{array}$} \\
\hline \begin{tabular}{|l|} 
Soil \\
Properties
\end{tabular} & $\begin{array}{l}\text { Slope } \\
\text { Angle }\end{array}$ & $\begin{array}{l}\text { Direct } \\
\text { Ascent }\end{array}$ & \begin{tabular}{|l|} 
Body \\
Leveling \\
(25ªttack)
\end{tabular} & $\begin{array}{l}\text { Inch- } \\
\text { worm }\end{array}$ \\
\hline \multirow{2}{*}{$\begin{array}{l}\text { Low strength, } \\
\text { fine volcanic } \\
\text { basalt, } \\
\text { Mauna Kea }\end{array}$} & 15 & - & 10 & $F$ \\
\hline & 20 & - & 30 & \\
\hline \multirow{3}{*}{$\begin{array}{l}\text { Low strength, } \\
\text { fine sand, } \\
\text { Moses Lake }\end{array}$} & 10 & 22 & 4 & \\
\hline & 15 & 24 & 5 & \\
\hline & 20 & 100 & 65 & \\
\hline \multirow{3}{*}{$\begin{array}{l}\text { Med. strength, } \\
\text { fine sand, } \\
\text { Moses Lake }\end{array}$} & 10 & 8 & 2 & \\
\hline & 15 & 9 & 7 & 8 \\
\hline & 20 & 45 & 13 & 30 \\
\hline \multirow{3}{*}{$\begin{array}{l}\text { High strength, } \\
\text { fine sand, } \\
\text { Moses Lake }\end{array}$} & 10 & 6 & 1 & I. \\
\hline & 15 & 8 & 3 & - \\
\hline & 20 & 16 & 10 & - \\
\hline
\end{tabular}

The center of mass shifting (body roll or 'leaning') was shown to successfully increase the steepness of slopes ascendable. The experiments were conducted with a $25^{\circ}$ angle attack from the horizontal. This value was determined experimentally to have adequate uphill progress and low slip. It was shown that with the transformable suspension of the Scarab rover, slopes of $20^{\circ}$ loose, dry, volcanic ash can be ascended under low risk.

\subsection{Autonomous Navigation}

Scarab navigates autonomously to sampling sites. Its goal is specified as a position with a radius (typically $10 \mathrm{~m}$ ) to define the area that it is to reach. For distant goals, a higher level process generates intermediate goals (typically with $50 \mathrm{~m}$ spacing). The operator may specify multiple goals, Scarab will reach each goal (and automatically generated intermediate goals) in order.

Scarab periodically scans the terrain using a TriDAR scanning laser rangefinder developed by Neptec Design Group. [Neptec08] Scarab acquires a scan after signficantly driving (3 to 4 meters) or turning (10 degrees) or after time has elapsed (100 seconds). The sensor is used to acquire a dense scan and takes several seconds, so Scarab must stop to avoid warping the data. The navigation software assumes a static world, meaning the terrain does not change in between scans.

Each scan is incorporated into a single world model. The scan generates a point cloud, which a collection of points on the surface of the terrain. First this data is filtered (to remove noise and artifacts) and

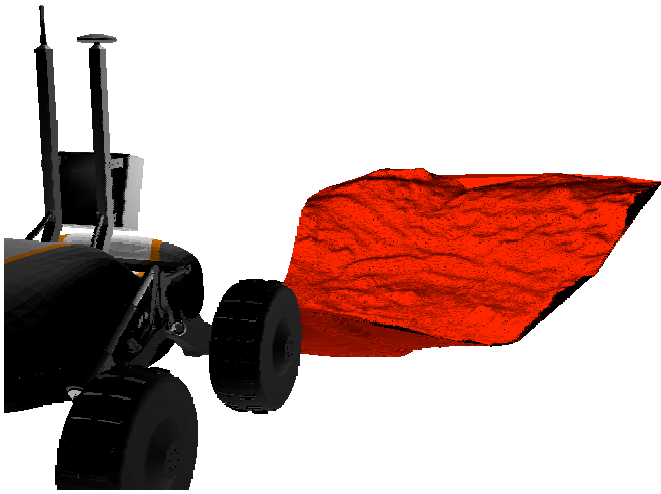

Figure 8. Dense laser scan is acquired ahead of the robot and this range data is used to produce a surface model of the terrain.

transformed into the vehicle's coordinate frame. Next Scarab performs a coarse data reduction on the point cloud and generates a mesh. The mesh is then further reduced to eliminate redundant data. Finally that mesh is merged with prior data to generate the terrain model that is used to identify obstacles and select the best path to the goal. Many candidate vehicle motions are evaluated in the near- and farfield. The nearfield portion involves simulating vehicle motion on the mesh to identify collision and slope hazards. The farfield portion uses a heuristic to estimate how much progress each motion made to the goal. A weighted average is used to combine safety in the nearfield with progress in the farfield.

Our experimental approach has been to conduct 1 $\mathrm{km}$ traverses in a variety of terrains with progressive improvements to algorithms and software. At both

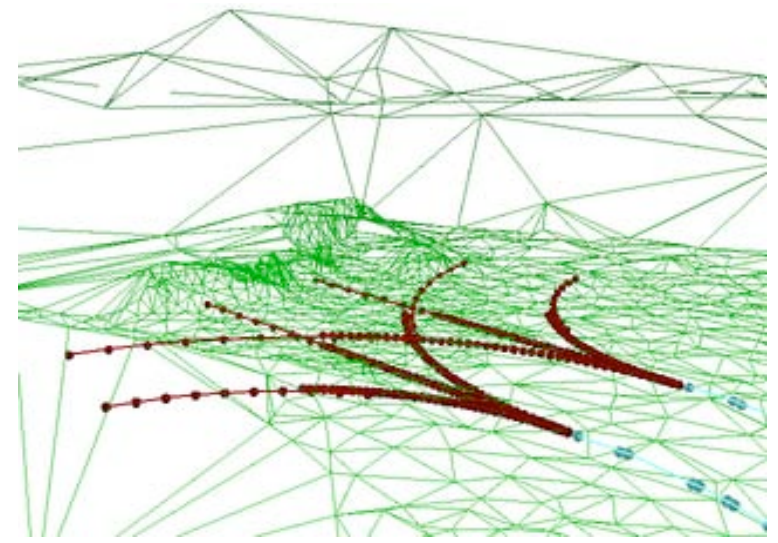

Figure 9. The terrain model is used to evaluate and refine the rover path. Modeling and then evaluation iterate to navigate to the goal.

the Moses Lake and Mauna Kea sites, Scarab autonomously completed the following objectives; travel over $3 \mathrm{~km}$, perform 2 night traverses and simulate crater descent. Night traverses are kilometer scale and performed after sunset, they account for 


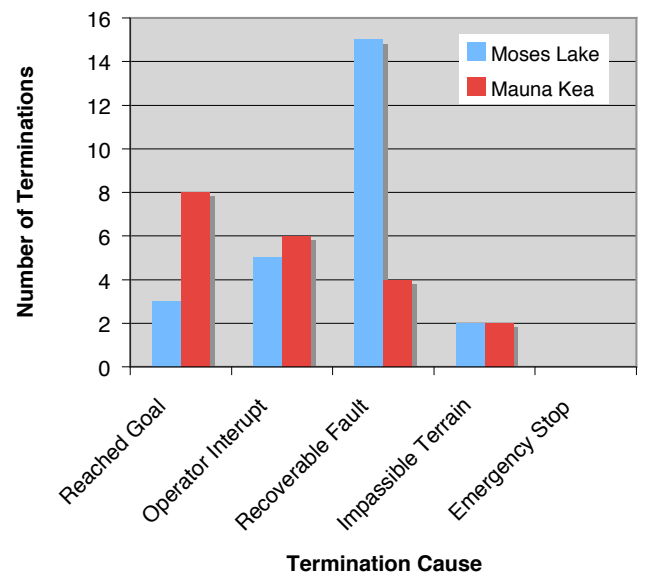

\section{Chart 1. Termination conditions in autonomous navigation experiments.}

most of the total distance at each site. Crater descent was simulated with a long $(100 \mathrm{~m})$ traverse that includes descending a steep $\left(10^{\circ}\right)$ slope.

Scarab completed a total of $3.6 \mathrm{~km}$ over 27 runs at Moses Lake. The first dark traverse was $1.2 \mathrm{~km}$ with 4 interventions due to sensor faults and one due to a motor controller error. Both of these types of faults are recoverable; they do not jeopardize the rover and were easily solved by power-cycling a single device. A second overnight traverse used an alternative navigation algorithm and completed $1.1 \mathrm{~km}$ with two interventions due to localization error.

Scarab traveled $3.0 \mathrm{~km}$ over 20 runs at Mauna Kea, most of this was accomplished during the two overnight traverses. The first dark traverse was split into two runs; after 199m the traverse was paused for programatic reasons, later Scarab resumed and completed an additional $779 \mathrm{~m}$ before stopping due to a software error. The second overnight traverse was also split in two; the first run was $312 \mathrm{~m}$ and stopped on a software error, the second was $989 \mathrm{~m}$ and ended with a CANBus fault. All of these errors are recoverable.

At each site, Scarab autonomously completed a simulated crater descent using available analog terrain. At Moses Lake, Scarab drove into a $9 \mathrm{~m}$ pit with 10-20 degree sloped sides. This was safely accomplished with two undirected switchback maneuvers. At Mauna Kea, Scarab repeatedly drove down a winding drainage channel. The route was over $100 \mathrm{~m}$ long and descended over $25 \mathrm{~m}$ with an average grade of about $10^{\circ}$. This descent was part of both overnight traverses as well as a third unrelated demonstration.

Traverse termination conditions for both field tests are shown in Chart 1. No interventions were required to stop the vehicle from driving into a hazard. At Moses Lake, most traverses (15 of 25) ended with a recoverable fault. On Mauna Kea the navigation method had improved and most traverses ended by reaching the goal ( 8 of 20 ) or stopping the traverse for other reasons (6 of 20). Recoverable faults are problems that were solved by power-cycling a device or updating a software module.

\section{Conclusion}

The Scarab rover has been uniquely configured for the transport and stabilization of a 1-meter coring drill and associated soil analysis instruments. The benefits of central-mounting and active body height and roll control are apparent in deployment of the drill and improved ability to climb cross-slope.

Field experimentation has quantified drawbar pull and slope climbing ability as well as power required for these activities under a variety of soil and terrain conditions. Field demonstrations have also proven the capability of the laser-based navigation system for kilometer-scale autonomous traverse.

\section{Acknowledgments}

This paper describes the collective efforts of the Lunar Rover Initiative at Carnegie Mellon. We gratefully acknowledge the contributions of our technical team and the assistance of Phillipe Ayoub, Paul Bartlett, John Thornton, Chuck Whittaker, and William Whittaker. This research was supported by NASA under grants, NNX07AE30G, John Caruso, Program Scientist, and NNX08AJ99G, Robert Ambrose, Program Scientist.

\section{References}

[Bartlett08] Bartlett, P., Wettergreen, D., and Whittaker, W., "Design of the Scarab Rover for Mobility and Drilling in Lunar Cold Traps," International Symposium on Artificial Intelligence, Robotics and Automation in Space (iSAIRAS), Los Angeles, February 2008.

[Harrison08] D.A. Harrison, R. Ambrose, B. Bluethmann, and L. Junkin, "Next Generation Rover for Lunar Exploration," JSC released document, IEEEAC paper 1196, 2008

[Kleinhenz09] Kleinhenz, J., et al., "Development of a reactor for the extraction of oxygen and volatiles from lunar regolith," AIAA Aerospace Sciences, Orlando, January 2009.

[Muscatello09] Muscatello, A., et al., "Lunar Water Resource Demonstration," AIAA Aerospace Sciences, Orlando, January 2009.

[Neptec08] http://www.neptec.com/Neptec_TriDAR.html [Norcat08] http://www.norcat.org/innovation-space.aspx [Sanders09], Sanders, G., et al., "In Situ Resource

Utilization (ISRU) Program," AIAA Aerospace Sciences, Orlando, January 2009.

[Spudis06] Spudis, P., "Ice on the Moon", The Space Review, November 2006. 